\title{
Implications of solar flare hard X-ray "knee" spectra observed by RHESSI
}

\author{
A. J. Conway ${ }^{1}$, J. C. Brown ${ }^{2}$, B. A. C. Eves ${ }^{1}$, and E. Kontar ${ }^{2}$ \\ ${ }^{1}$ Dept. of Physics \& Astronomy, The Open University, Milton Keynes MK7 6AA, UK \\ e-mail: b.a.c.eves@open.ac.uk \\ 2 Dept. of Physics \& Astronomy, University of Glasgow, Glasgow G12 8QQ, UK \\ e-mail: john@astro.gla.ac.uk, eduard@astro.gla.ac.uk \\ Received 10 March 2003 / Accepted 12 June 2003
}

\begin{abstract}
We analyse the RHESSI photon spectra of four flares that exhibit significant deviations from power laws - i.e. changes in the "local" Hard X-ray spectral index. These spectra are characterised by two regions of constant power law index connected by a region of changing spectral index - the "knee". We develop theoretical and numerical methods of describing such knees in terms of variable photon spectral indices and we study the results of their inversions for source mean thin target and collisional thick target injection electron spectra. We show that a particularly sharp knee can produce unphysical negative values in the electron spectra, and we derive inequalities that can be used to test for this without the need for an inversion to be performed. Such unphysical features would indicate that source model assumptions were being violated, particularly strongly for the collisional thick target model which assumes a specific form for electron energy loss. For all four flares considered here we find that the knees do not correspond to unphysical electron spectra. In the three flares that have downward knees we conclude that the knee can be explained in terms of transport effects through a region of non-uniform ionisation. In the other flare, which has an upward knee, we conclude that it is most likely a feature of the accelerated spectrum.
\end{abstract}

Key words. Sun: X-rays, gamma rays - Sun: flares

\section{Introduction}

Solar flare hard X-ray $(\geq 10 \mathrm{keV})$ spectra have long been recognised (e.g. Brown 1971; Lin \& Hudson 1976) as important diagnostics of acceleration mechanisms for electrons and of their substantial role in impulsive flare energy transport. A feature present in many HXR spectra is a downward bend toward higher spectral index $(\gamma=-\mathrm{d} \ln J / \mathrm{d} \ln \epsilon)$ at increasing photon energy. Such bends, or "knees", were recorded earlier even with low resolution instruments and the first high resolution spectrum (Lin \& Schwartz 1987) indicated that the knee was fairly sharp. First data from the RHESSI mission (Lin \& Dennis 2002) confirm that many flares do not conform to a single power law in the $20-100 \mathrm{keV}$ photon energy range and the $1 \mathrm{keV}$ energy resolution now permits detailed investigation of spectral "knees". Since the photon spectrum $I(\epsilon)$ is an integral of the inverse mean electron spectrum $\bar{F}(E)$ convoluted with the "wide" bremsstrahlung cross-section, abrupt knees in $I(\epsilon)$ imply very sharp features in $\bar{F}(E)$ (which is essentially the derivative of $I(\epsilon)$ ). In the case of the thick target model (Brown 1971), $\bar{F}(E)$ results from the further convolution of the "injection spectrum" $F_{0}\left(E_{0}\right)$ with the energy loss rate along the electron stopping path. Then the implied $F_{0}\left(E_{0}\right)$ has to have extremely sharp corresponding structures (since it is essentially the second derivative of $I(\epsilon))$.

In this paper we address the implications of RHESSI spectral knee data, building on previous work on the problem. In

Send offprint requests to: A. J. Conway, e-mail: a.j.conway@open .ac .uk particular, Brown (1971) first recognised the interpretation of HXR spectra as an inverse problem and obtained an analytical inversion for the Bethe-Heitler cross-section for thin target and collisional thick target cases. Brown \& MacKinnon (1985) discussed analytically the effects of generalised energy loss functions for thick target spectra and specific cases were considered by Brown et al. (1998), Haydock et al. (2001), Emslie et al. (2001). Brown \& Emslie (1987) discussed analytic restrictions on the properties of $I(\epsilon)$ for them to be interpretable in terms of thin, thick, and thermal models, particularly in the case of the Kramers cross-section approximation (their findings being generalised to other cross-sections in Brown et al. 2003). Thompson et al. (1992), Johns \& Lin (1992), Piana et al. (1995), Piana \& Brown (1998), McArthur (2000) have carried out numerical inversions of the first real high resolution data (Lin \& Schwartz 1987) for thin and thick target models and Piana et al. (2001) for a thermal model with a distribution of temperatures. The specific issue of spectral knees, their sharpness, and their relation to features in the source and injection electron spectra was first discussed by Brown et al. (1991).

In Sect. 2 we outline the mathematical framework of how electron and photon spectra are related, noting in particular how any feature in the photon spectrum must correspond to a sharper feature in the electron spectrum. In Sect. 3 we develop the theory to deal with knee spectra in the Kramer's crosssection approximation and then go on in Sect. 4 to apply this theory in interpreting spectra observed by RHESSI. Section 5 discusses the results and their implications for electron acceleration and transport in solar flares. 


\section{Basic theory}

\subsection{Source average equivalent thin target electron spectra}

Here we wish to relate the observed photon spectrum $I(\epsilon)$ (number of photons $\mathrm{cm}^{-2} \mathrm{keV}^{-1}$ ) to the electron spectrum $\bar{F}(E)$ (flux of electrons $\mathrm{cm}^{-2} \mathrm{keV}^{-1}$ ) averaged over the flaring region. For an ion-summed electron-ion bremsstrahlung crosssection $Q(\epsilon, E)$, differential in photon energy $\epsilon$, at electron en$\operatorname{ergy} E$, written as

$Q(\epsilon, E)=\frac{Q_{0} m_{\mathrm{e}} c^{2}}{\epsilon E} q(\epsilon, E)$.

The optically thin photon flux spectrum $I(\epsilon)$ at a distance $D$, can always be written as (Brown 1971), neglecting all the effects except collisions,

$I(\epsilon)=\frac{Q_{0} m_{\mathrm{e}} c^{2}}{\epsilon} \frac{\bar{n}_{\mathrm{p}} V}{4 \pi D^{2}} \int_{\epsilon}^{\infty} \frac{\bar{F}(E)}{E} q(\epsilon, E) \mathrm{d} E$

where the averages are defined over the flaring volume:

$\overline{n_{\mathrm{p}}}=\frac{1}{V} \int_{V} n_{\mathrm{p}}(r) \mathrm{d} V \quad \bar{F}(E)=\frac{1}{\bar{n}_{\mathrm{p}} V} \int_{V} F(E, r) n_{\mathrm{p}}(r) \mathrm{d} V$

where $n_{\mathrm{p}}(r)$ is the density and $F(E, r)$ is the electron flux spectrum at point $r$ within the flaring volume $V$. Note (cf. Brown $\&$ Emslie 1987) that $\bar{F}(\mathrm{E})$ can always be defined for any source model - Brown et al. (2003).

For mathematical convenience we will work with the normalised spectrum $h(\epsilon)$ defined so as to remove all non-energy dependent factors in (2)

$h(\epsilon)=\epsilon I(\epsilon) \frac{4 \pi D^{2}}{Q_{0} m_{\mathrm{e}} c^{2} \bar{n}_{\mathrm{p}} V}=\int_{\epsilon}^{\infty} \frac{\bar{F}(E)}{E} q(\epsilon, E) \mathrm{d} E$.

So, given knowledge of $q(\epsilon, E)$, we can obtain the electron flux spectrum from $h(\epsilon)$ as long as the integral equation solution exists.

\subsection{Thick target with general energy losses}

Here we wish to relate the injected electron flux spectrum $F_{0}\left(E_{0}\right)$ to the observed photon flux spectrum $I(\epsilon)$. To do this a restrictive model interpretation is introduced that $\bar{F}(E)$ arises by "injection" of a flux spectrum $F_{0}\left(E_{0}\right)$ of electrons of initial energies $E_{0}$ and that all these electrons stop in a "thick" target region in times shorter than the data integration time, emitting bremsstrahlung at $\epsilon$ until $E_{0} \leq \epsilon$. It is also generally assumed that the energy loss rate per unit column density $N$ for an electron of energy $E$ is the same for all electrons of the same $E_{0}$ and so in general can be written as (Brown \& MacKinnon 1985)

$\frac{\mathrm{d} E}{\mathrm{~d} N}=-R(E) \frac{K}{E}$

where $N$ is the plasma column density $\int_{0}^{S} n_{\mathrm{p}} \mathrm{d} s$ along the electron path, $K=2 \pi e^{4} \Lambda$, and $\mathrm{R}(\mathrm{E})$ is the ratio of energy loss rate to the collisional rate $K / E$ in a fully ionised target (cf. Brown 1971; Emslie 1978). Here we discuss only the collisional case $R(E)=1$ Then one can write, for injection over area $A$ (cf. Brown 1971)

$I(\epsilon)=\frac{Q_{0} m_{\mathrm{e}} c^{2} A}{4 \pi D^{2} K \epsilon} \int_{\epsilon}^{\infty} q(\epsilon, E) \int_{E}^{\infty} F_{0}\left(E_{0}\right) \mathrm{d} E_{0} \mathrm{~d} E$.

Comparing (2) and (6) we see that, for the thick target, $\bar{F}(E)$ is related to $F_{0}\left(E_{0}\right)$ for the same $I(\epsilon)$ (Brown \& Emslie 1987) by

$\bar{n}_{\mathrm{p}} V \bar{F}(E)=\frac{A E}{K} \int_{E}^{\infty} F_{0}\left(E_{0}\right) \mathrm{d} E_{0}$

so that the injected spectrum can be related to derivative of the thin target spectrum according to

$F_{0}\left(E_{0}\right)=\frac{K \bar{n}_{\mathrm{p}} V}{A}\left[-\frac{\mathrm{d}}{\mathrm{d} E}\left(\frac{\bar{F}(E)}{E}\right)\right]_{E=E_{0}}$.

Note that this relationship is independent of $q(\epsilon, E)$.

\subsection{Kramer's cross-section}

The Kramer's approximation means that $q(\epsilon, E)=1$ for $E>\epsilon$ and $q(\epsilon, E)=0$ for $E<\epsilon$. Under this assumption (4) leads to a simple relationship between the (normalised) observed spectrum $h(\epsilon)$ and the averaged photon flux $\bar{F}(E)$ :

$\bar{F}(E)=-\left[\epsilon \frac{\mathrm{d} h}{\mathrm{~d} \epsilon}\right]_{\epsilon=E}$

and we can use (6) with $R(E)=1$ to write the injected spectra as (remembering the restrictive thick target assumptions; Brown 1971)

$F_{0}\left(E_{0}\right)=\frac{K n_{p} V}{A}\left[\frac{\mathrm{d}^{2} \mathrm{~h}}{\mathrm{~d} \epsilon^{2}}\right]_{\epsilon=E_{0}}$.

The rigorous mathematical implications of a more realistic cross-section are beyond the scope of this paper. However, it is important to realise that the finite discontinuity in the Kramer's cross-section at $E=\epsilon$ cannot be the sole cause of sharp features in the inverted electron spectrum discussed in this paper. This is supported by the fact that electron spectra with sharp knee features lead to very similar photon spectra irrespective of whether Kramer's or the Bethe-Heitler cross-section, which has no such discontinuity, are used. It is also worth noting that, as pointed about by Johns \& Lin (1992), more accurate crosssections also have a finite discontinuity at $E=\epsilon$.

\section{Knee spectra theory}

\subsection{Definitions}

Without any loss of generality, any photon spectrum $H(\epsilon)$ can be written in the form

$h(\epsilon)=h_{*}\left(\frac{\epsilon}{\epsilon_{*}}\right)^{-\gamma(\epsilon)}$

where $\epsilon_{*}$ is a knee energy parameter. Note that the form of $\gamma(\epsilon)$ in general depends on our choice of $\epsilon_{*}$. We choose to define 
the knee energy as that where the rate of change of spectral in$\operatorname{dex}(|\mathrm{d} \gamma / \mathrm{d} \epsilon|)$ is maximum, which in turn means that the second derivative must be zero $\left(\mathrm{d}^{2} \gamma / \mathrm{d} \epsilon^{2}=0\right)$. It is important to realise the variable spectral index $\gamma(\epsilon)$ is not equal to the gradient of the spectrum on a $\log$-log plot - i.e. $\gamma(\epsilon)=-\mathrm{d} \log I / \mathrm{d} \log \epsilon$ unless the gradient is constant. The relationship between log-log gradient and $\gamma$ is discussed further in the Appendix along with methods of estimating them from discrete spectra with noise.

We need not be concerned with the precise meaning of $\gamma(\epsilon)$ other than its use in defining two properties of a spectra knee: its size $\Delta \gamma$ and its width $\Delta \epsilon$. For the spectra of interest here we can define these properties using a smooth parametric form for $\gamma(\epsilon)$ :

$\gamma(\epsilon)=\gamma_{1}+\frac{\Delta \gamma}{1+\mathrm{e}^{-\left(\epsilon-\epsilon_{*}\right) / \Delta \epsilon}}$.

This form has the following properties if $\Delta \epsilon$ is much smaller than the energies of interest: $\gamma(0) \approx \gamma_{1}, \gamma(\infty)=\gamma_{2}$, and $\gamma_{*}=\gamma\left(\epsilon_{*}\right)=\left(\gamma_{1}+\gamma_{2}\right) / 2$. It also satisfies the above derivative definitions of the knee energy. In working with observed spectra, we will use (11) together with (12) to find the set of parameters $\gamma_{1}, \gamma_{2}, \Delta \epsilon$ and $h_{*}$ that minimize the following reduced $\chi^{2}$ on a set of $M$ energy bins

$\chi^{2}=\frac{1}{M-4} \sum_{i=1}^{M} \frac{\left[H_{i}-h\left(\epsilon_{i}\right)\right]^{2}}{\sigma_{i}^{2}}$.

For spectra considered in this paper we used $1 \mathrm{keV}$-wide energy bins between $20 \mathrm{keV}$ and $100 \mathrm{keV}$ (i.e. $M=80$ ) to evaluate our $\chi^{2}$. In every case $\chi^{2} \sim 1$ was achieved. (Problems introduced by thermal components in the spectrum were avoided by using a minimum energy of $20 \mathrm{keV}$, but they still prevented $\chi^{2}$ from coming as close to unity as a degrees of freedom of $M-4=76$ would suggest.)

\subsection{Thin target}

The thin target solution for $\bar{F}(E)$ (9) given the definition of the variable spectral index spectrum (11) (we are not yet assuming a specific form of $\gamma$ ) is

$\bar{F}(E)=h(E) \gamma(E)\left[1+\ln \left(\frac{\epsilon}{\epsilon_{*}}\right) \frac{\mathrm{d} \ln \gamma}{\mathrm{d} \ln \epsilon}\right]_{\epsilon=E}$.

Away from the knee, where $\gamma$ tends towards constant values, this solution reproduces the well-known thin target formula results: the electron flux spectrum $F(E)$ has the same index as the photon energy spectrum, $h(\epsilon) \propto \epsilon I(\epsilon)$. We now wish to examine (14) for unphysical behaviour namely where $\bar{F}(E)$ becomes negative as a result of variations in spectral index. Negativity problems occur when the following inequality is true

$\ln \left(\frac{\epsilon}{\epsilon_{*}}\right) \frac{\mathrm{d} \ln \gamma}{\mathrm{d} \ln \epsilon}<-1$.

This will occur in the following three cases: 1) increasing $\gamma$ below the knee energy, 2) decreasing gamma above the knee energy 3 ) the log-log derivative of $\gamma$ goes to $-\infty$ "faster" than the logarithm goes to zero at the knee energy. Since the logarithm factor goes to zero at the knee energy, cases 1 and 2 require that the spectral indices vary significantly away from the knee energy. Case 3 is only a problem for knees that are infinitely sharp (a discontinuity of the first kind), which cannot occur in reality. (Mathematically the knee needs to be not only infinitely sharp but be so in particular way. Specifically, writing $\epsilon=\epsilon_{*}+\delta \epsilon$, for small $\delta \epsilon$ the derivative $\mathrm{d} \ln \gamma / \mathrm{d} \ln \epsilon$ must have a term in $(\delta \epsilon)^{-n}$ where $n>1$.) So, in summary, we expect that the thin target expression for $\bar{F}(E)$ in (14) will be physical for cases where variation in spectral index is localised to a knee, even for very sharp knees.

\subsection{Collisional thick target}

For the thick target model $(R(E)=1),(10)$ with expression $(11)$ gives

$F_{0}\left(E_{0}\right)=\frac{K \bar{n}_{\mathrm{p}} V}{A} h\left(E_{0}\right) g\left(E_{0}\right)$

where

$$
\begin{aligned}
g\left(E_{0}\right)= & {\left[\frac{\gamma(\gamma+1)}{\epsilon^{2}}+\frac{2}{\epsilon}\left(\gamma \ln \frac{\epsilon}{\epsilon_{*}}-1\right) \frac{\mathrm{d} \gamma}{\mathrm{d} \epsilon}\right.} \\
& \left.+\left(\ln \frac{\epsilon}{\epsilon_{*}}\right)^{2}\left(\frac{\mathrm{d} \gamma}{\mathrm{d} \epsilon}\right)^{2}-\left(\ln \frac{\epsilon}{\epsilon_{*}}\right) \frac{\mathrm{d}^{2} \gamma}{\mathrm{d} \epsilon^{2}}\right]_{\epsilon=E_{0}}
\end{aligned}
$$

For photon energies far from the knee the first and second order derivatives of $\gamma$ tend to zero and the above electron spectrum tends towards the standard thick target results: the injected electron spectrum has a power law index of $\gamma+2$. We now turn our attention to cases where $F_{0}\left(E_{0}\right)$ is unphysical at the knee energy, specifically where $g\left(E_{0}\right)<0$. At the knee energy, by definition, the logarithm and the second derivative is zero, so we find that for $\epsilon=\epsilon_{*}$

$g\left(\epsilon_{*}\right)=\frac{\gamma\left(\epsilon_{0}\right)}{\epsilon_{*}^{2}}\left[(\gamma+1)-2 \frac{\mathrm{d} \ln \gamma}{\mathrm{d} \ln \epsilon}\right]_{\epsilon=\epsilon_{*}}$.

In contrast with the thin target case 3 , the $\mathrm{d} \gamma / \mathrm{d} \epsilon$ term is nonzero at the knee so a large rate of change in $\gamma(\epsilon)$ results in a large contribution to $F_{0}\left(E_{0}=\epsilon_{*}\right)$. The contribution is positive for an upward knee (decreasing $\gamma$ ) and negative for a downward knee (increasing $\gamma$ ). In the latter case, if $\gamma$ increases rapidly enough, $g\left(E_{0}\right)$ can become negative around $E_{0}=\epsilon_{*}$ and so give an unphysical $F_{0}\left(E_{0}\right)$. Specifically, the condition on $\gamma(\epsilon)$ for this to happen is, by (17)

$\frac{\mathrm{d} \ln \gamma}{\mathrm{d} \ln \epsilon}>\frac{\gamma+1}{2}$

Using the parametric form for $\gamma(\epsilon)$ (12) we can derive the following inequality which, if true, tells us that the thick target spectrum $F_{0}\left(E_{0}\right)$ has become negative:

$\frac{\Delta \epsilon}{\epsilon_{*}}<\frac{\Delta \gamma}{2 \gamma_{*}\left(\gamma_{*}+1\right)}$

On this basis, a spectrum with a knee at $50 \mathrm{keV}$ which steepens from $\gamma_{1}=3$ to $\gamma_{2}=5$ must have a $\Delta \epsilon$ greater than $2.5 \mathrm{keV}$ to ensure that $F_{0}$ is positive. The results of this section generalise those of Brown et al. (1991) for a special case. 
Table 1. Properties of analysed knee flares.

\begin{tabular}{lllll}
\hline \hline & $20 / 02 / 2002$ & $20 / 02 / 2002$ & $17 / 03 / 2002$ & $06 / 08 / 2002$ \\
\hline Time interval & $0957: 33.41-$ & $1105: 56.9-$ & $1928: 08-$ & $1256: 24: 00-$ \\
& $0958: 21 . .04$ & $1106: 35.9$ & $1928: 57$ & $1257: 40$ \\
\hline GOES CLASS & M4.3 & C7.5 & M4.0 & C7.9 \\
\hline Emission measure & N/A & 0.1159 & 0.57 & 0.031 \\
$\left(10^{49} \mathrm{~cm}^{-3}\right)$ & & & & \\
\hline $\begin{array}{l}\text { Plasma temperature } \\
k T(\mathrm{keV})\end{array}$ & $\mathrm{N} / \mathrm{A}$ & 1.568 & 1.45 & 1.56 \\
\hline $\begin{array}{l}\text { Knee photon flux } H_{*} \\
\left(\mathrm{~s}^{-1} \mathrm{~cm}^{-2} \mathrm{keV} \mathrm{V}^{-1}\right)\end{array}$ & 0.52 & 0.48 & 2.7 & 0.83 \\
\hline$\gamma_{1}$ & 7.4 & 3.5 & 3.5 & 3.6 \\
\hline Knee energy $\epsilon_{*}$ & 37 & 49 & 44 & 36 \\
\hline$\gamma_{2}$ & 3.9 & 4.2 & 4.3 & 4.3 \\
\hline$\Delta \gamma$ & -3.5 & 0.7 & 0.8 & 0.7 \\
\hline$\Delta \epsilon(\mathrm{keV})$ & 3 & 4 & 18 & 12 \\
\hline$\Delta \epsilon_{\text {crit }}(\mathrm{keV})$ & - & 1.2 & 1.1 & 0.86 \\
\hline Energy where Flare $=$ & & & & \\
Background/2 $(\mathrm{keV})$ & 57 & 52 & 81 & 47 \\
\hline Energy where Flare $=$ & & & & \\
Background/10 $(\mathrm{keV})$ & 45 & & & \\
\hline
\end{tabular}

\section{Application to RHESSI data}

\subsection{RHESSI spectra and $\gamma$ profiles}

RHESSI (Lin \& Dennis 2002) has nine detectors that individually record the arrival times and energies of incident photons. These data are relayed to the ground allowing choices of binning strategy in time and photon energy to be made during the analysis. For this work we required RHESSI's highest resolution of $1 \mathrm{keV}$ bins in the $10-100 \mathrm{keV}$ range, which forced us to omit data from detectors 2 and 7 because they have a poorer resolution than intended (Smith et al. 2002). We chose time bins equal to RHESSI's rotation period (as given for the time of the flare) because this ensured that there would be no modulation from the imaging grids. Before spectral analysis can be performed it is necessary to correct the data for the following effects: detector energy response, detector livetime, attenuator transmission, and imaging grid transmission and pulse pile-up (see below). These steps were performed by standard software using the most up to date information on the instrumental calibration (as of 21 February 2003). We then subtracted the background by using the SPEX package to interpolate between two background intervals, one before and one after the flare.

Figures 2 to 4 show the spectra of four flares that exhibit knees. For each spectra (in panel b) we present two versions of the $\gamma$ profile. To construct these we first used values of $\gamma_{1}, \gamma_{2}$, $\epsilon_{*}$ and $H_{*}$ from the SPEX fitting of the flare spectrum with a thermal plus double power law model. These values were then used as the starting point in fitting the parametric model for $\gamma$ (12) for parameters $\gamma_{1}, \gamma_{2}, H_{*}$ and $\Delta \epsilon$. Using only $H_{*}$ and $\epsilon_{*}$ we could then construct the $\gamma$ values and their error bars for each energy bin according to the method described in the Appendix.
A summary of flare properties is given in Table 1. The emission measure, plasma temperature $k T$ and knee energy $\epsilon_{*}$ are obtained from the SPEX thermal plus double power law fit. The values of $H_{*}$ and $\Delta \epsilon$ are from the fitting of the parametric form of $\gamma$. The last two rows give the energy at which the background-subtracted flare spectrum is equal to twice and ten times the background spectrum (the background spectrum is always very hard compared to the flare spectrum). For the two largest flares in terms of GOES class, 20 Feb. 020958 and 17 Mar. 02 1728, we have applied a correction for pulse pile up correction to their HXR spectra. The difference between pileup corrected and uncorrected spectra was slight and, for the purposes of this paper, made no significant differences to the properties quoted in the table (for example the power indices were unchanged and the knee energy was altered by $<1 \mathrm{keV}$ ).

\subsubsection{Feb. 2002, 0958 UT}

This flare's spectrum is very steep (index 7) until $40 \mathrm{keV}$ at which there is a very pronounced upward knee. This is in contrast to the other three flares presented here that all have downward knees. Despite being a reasonably sized flare in overall counts and despite hardening (index $\sim 4$ ) at higher energies, the low energy softness means that the background does become significant over the upper few decades of energy, becoming equal to half the flare spectrum at $82 \mathrm{keV}$. Fortunately the circumstances of this flare allowed a very good background spectrum to be estimated from intervals in the surrounding minutes of the peak, especially for higher energies. With this confidence in the background spectrum we were able to verify that the knee cannot easily be explained by supposing that the background 


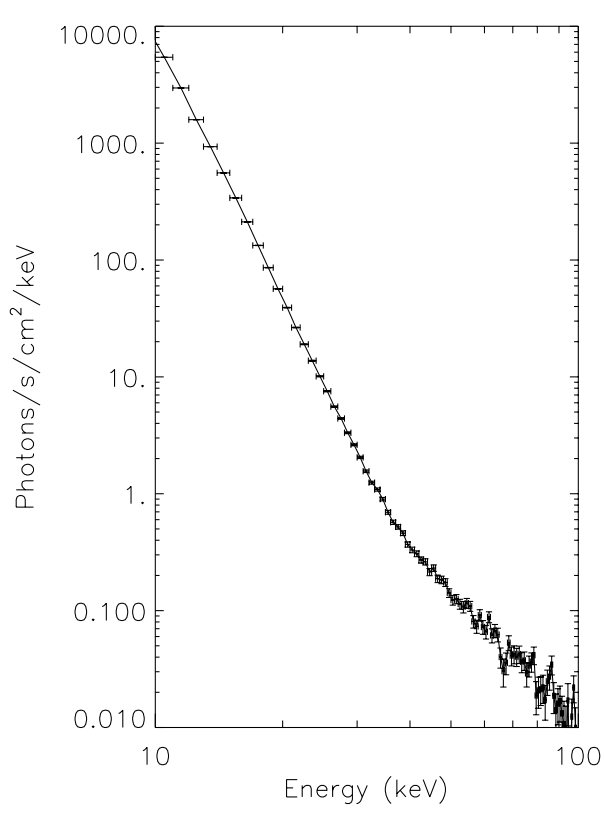

(a)

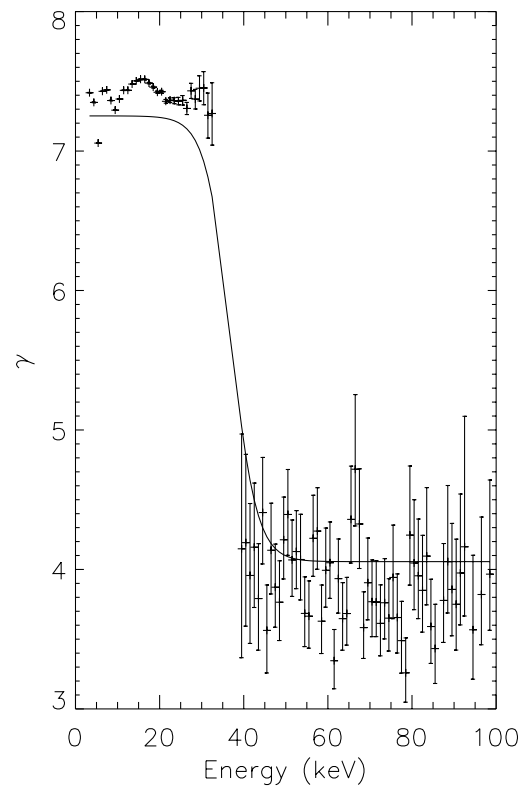

(b)

Fig. 1. a) The energy spectrum for the 20 February 0958 UT flare. b) The $\gamma$ profile: the points with error bars come from the logarithmic ratio method; the smooth curve shows the parametric fit; and the horizontal lines indicate the power indices obtained from the SPEX fitting. Points where error bars exceed the plot range are omitted.

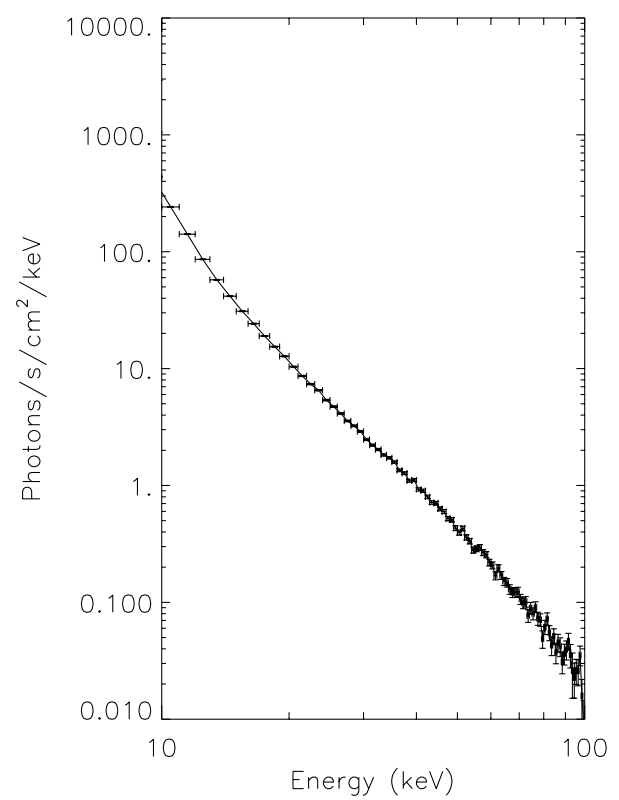

(a)

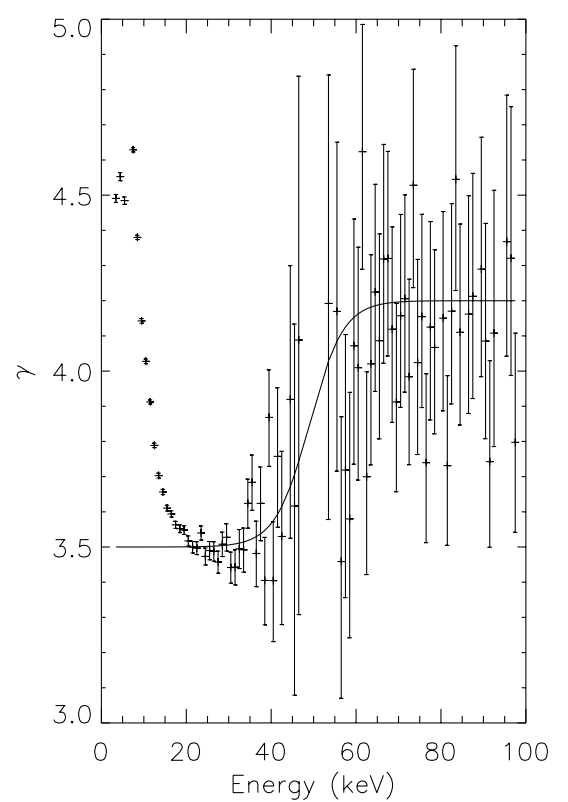

(b)

Fig. 2. a) The energy spectrum for the 20 February 1106 UT flare. b) The $\gamma$ profile.

spectrum is under-subtracted, which would produce hardening at higher energies. Firstly, the index of the background is much harder than this flare's $\gamma_{2}=4.2$ and the flare's $\gamma$ profile (Fig. 1b) shows little variation over this upper $60 \mathrm{keV}$ range. Since the addition of two power laws does not produce a power law, it would be hard to explain this profile as the flare plus some unsubtracted background. Secondly, although some residual line structure can be seen, it is comparable to the noise amplitude in size, and indicates, if anything, a slight over-subtraction because background lines look like downward pointing "absorption" lines. As a further test, we experimented with multiplying the background spectrum by a constant factor and subtracting it from the observed spectrum. Doing this with the restriction of keeping the background line prominence to the noise level could not "remove" the knee. We therefore conclude that the upward knee of this flare is a real feature of the flare and not a background artifact.

The other distinguishing feature of this flare is that there is virtually no thermal component; the $\gamma$ profile is quite flat compared to that of the other flares below $20 \mathrm{keV}$. The best double power law fit had $\chi^{2}=1.1$ whereas the best thermal plus power law fit had $\chi^{2}=3.3$. The absence or weakness 


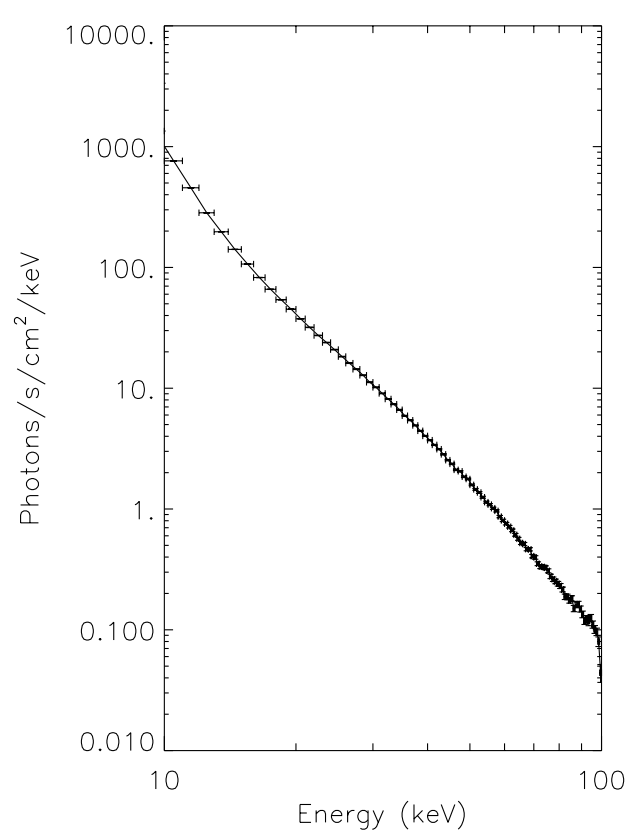

(a)

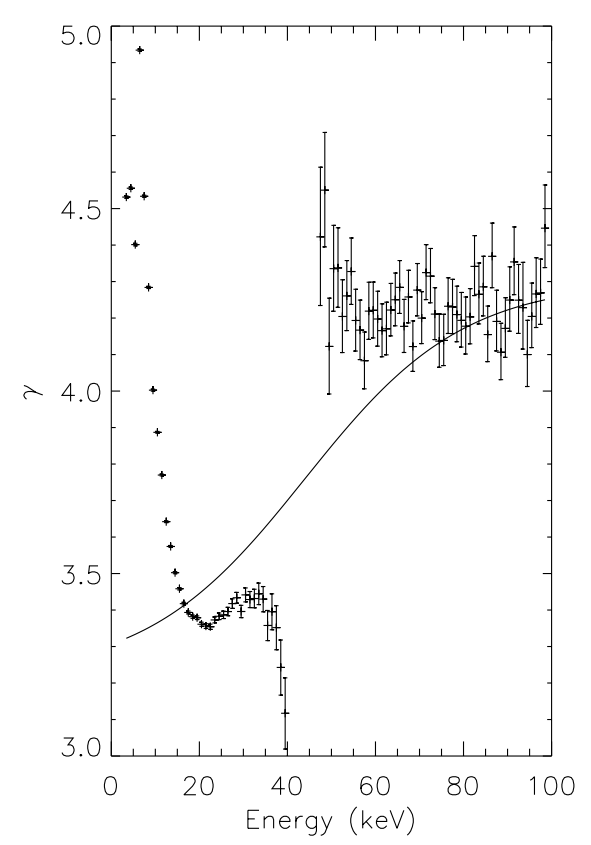

(b)

Fig. 3. a) The energy spectrum for the 17 March 1930 UT flare. b) The $\gamma$ profile.

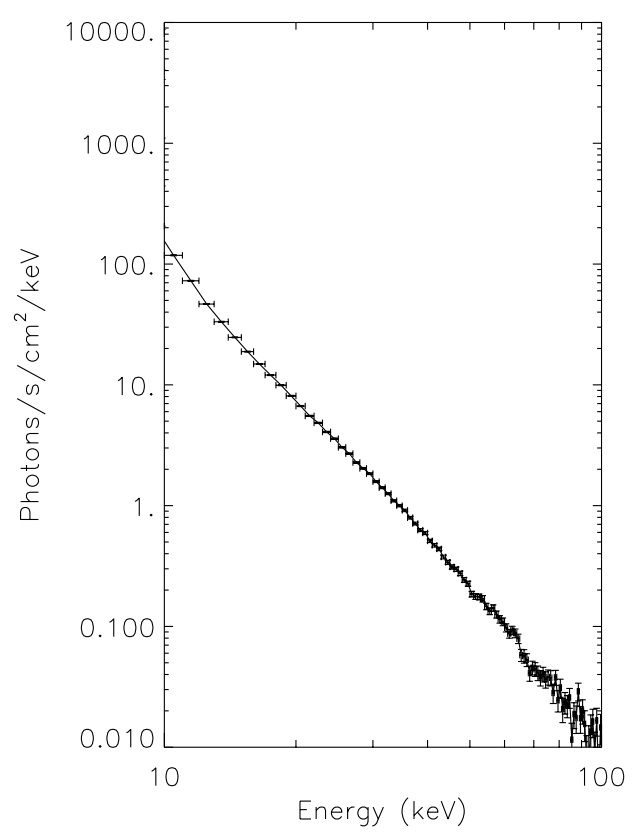

(a)

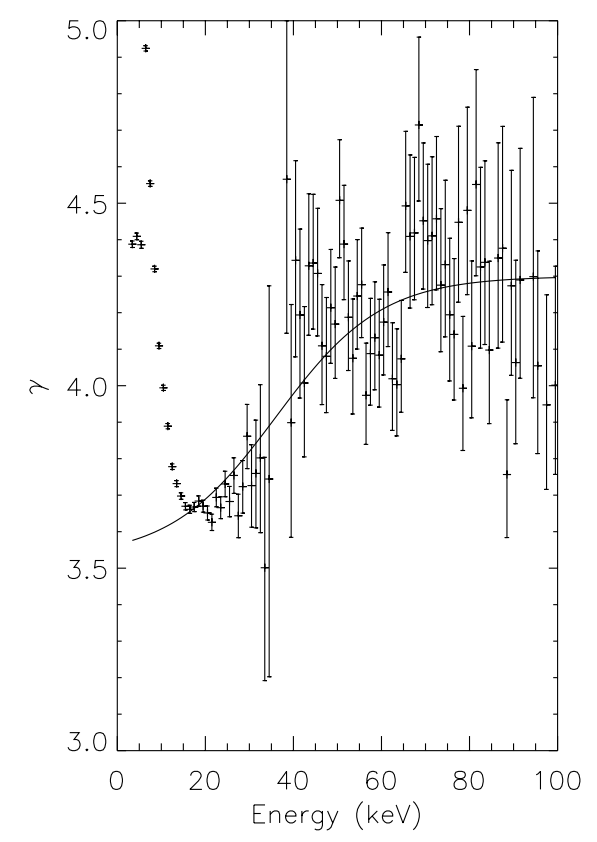

(b)

Fig. 4. a) The energy spectrum for the 6 August 1256 UT flare. b) The $\gamma$ profile.

of the thermal emission in this flare allows us to see that the spectral index of $\sim 7$ continues down to RHESSI's minimum observable energy of $3 \mathrm{keV}$ (shown on the $\gamma$ profile, but not the spectrum itself). Variations from this overall trend are apparent and are significant in terms of the error bars. The $\gamma$ profile clearly shows evidence of the iron lines below $10 \mathrm{keV}$ and of another sharp feature just above $10 \mathrm{keV}$, followed by a hump extending to about $20 \mathrm{keV}$. The spikes either side of the knee energy are an artifact of the logarithmic method of estimating $\gamma$ which is discussed in the Appendix.
Figure $1 \mathrm{~b}$ shows that there are small differences between the parametric and logarithmic ratio methods for estimating $\gamma$. This is surprising because both $\gamma$ profiles correspond to photon spectra that fit the observations to within given uncertainties. However, the fact that the ratio method's values are overestimates below the knee energy and underestimates above it suggest that the differences are due to the bias inherent in the ratio method (see the Appendix). This can be seen to some extent in the $\gamma$ profiles for other flares, though in those the effect of the thermal component is more pronounced. 


\subsubsection{Feb. 2002, 1106 UT}

This flare occurred near the limb and has attracted much attention due to its interesting spatial structure (Vilmer et al. 2002). The spectrum clearly shows a softening and a thermal component at low energies. The spectrum has a power law of index 3.5 from about $20 \mathrm{keV}$ until the knee just below $60 \mathrm{keV}$. Thereafter the index is approximately 4.3 , though noise becomes significant on the $\gamma$ profile above $60 \mathrm{keV}$.

The background subtraction in this case was problematic due to a brush with the edge of the SAA just before the flare. The effect was to cause an apparently decreasing background during the main peak of the flare. We therefore chose the before and after background intervals so that the linear interpolation would, at least in part, correct for the declining trend in the background. This SAA problem together with the fact that this is a small flare makes the background a more delicate issue than was the case for the 0958 flare. Specifically, the downward knee could be the result of too much background being subtracted. However, based on background subtraction tests similar to those performed on the 0958 UT flare we believe that the knee is a real feature of the flare. In fact, careful inspection of Fig. 2a shows that the high energy background lines are perhaps slightly under-subtracted.

\subsubsection{Mar. 2002, 1930 UT}

This flare is similar to the 20 Feb. 0958 flare in GOES class but by virtue of its harder spectrum it is a brighter hard X-ray flare. The constancy (within the noise) of $\gamma \sim 4.1$ is apparent at energies above about $45 \mathrm{keV}$, though the strong thermal component hides non-thermal emission below about $10 \mathrm{keV}$. From $10 \mathrm{keV}$ to $30 \mathrm{keV}$ there is a constant power law of index 3.2 , followed by a gradual softening leading up to the knee energy at $40 \mathrm{keV}$. The background is not significant with this flare as it is always more than a factor of ten less than the flare spectrum.

\subsubsection{Aug. 2002, 1256 UT}

This flare's spectrum is similar to the 17 Mar. 1930 flare, with a dominant thermal component below $10 \mathrm{keV}$, an initial index of 3.4 , and a knee at about $40 \mathrm{keV}$ leading to a slightly steeper index of 4.2. Despite the growing error bars from low energies towards the knee, there is a clear rising trend in $\gamma$ and no evidence of a region of constant index. If there is one, it is presumably less than 3.5 and completely masked by the strong thermal component. The background is not a problem with this flare as it is more than a factor of ten less than the flare at the knee.

\subsection{Inverted spectra}

Figures 5-8 show the inverted electron flux spectra for the four flares. The points on thin target spectra are obtained by taking the numerical derivative of the data $H_{i}$ according to (9). Likewise the points for the collisional thick target spectra are obtained from a double numerical derivative according to (10). Since these derivatives involve only differencing with no smoothing, i.e. $\mathrm{d} h / \mathrm{d} \epsilon \sim\left(H_{i+1}-H_{i}\right) /\left(\epsilon_{i+1}-\epsilon_{i}\right)$, the noise renders their results meaningless at higher energies. As an alternative we used the parametric fit for $\gamma$ in the analytic forms of the thin (14) and collisional thick (16) target to provide the curves plotted with the points. These spectra represented a highly smoothed form of inversion but would still show any unphysical behaviour (the spectra being negative) due to sharp knees. Clearly we can see no such behaviour in these plots within the data error limits. This can also be seen from the inequality condition on negativity in the collisional thick target electron spectra (19). It places the lower limits $\Delta \epsilon_{\text {crit }}$ given in Table 1 which are always much less than the $\Delta \epsilon$ for each flare (the 20 Feb. 020958 flare has no value because it has an upward knee).

Although there are no negativity problems, it is possible to see that the knee in photon spectrum corresponds to more complex features in the knee of the collisional thick target spectra. For example, in the 20 Feb. 020958 flare (Fig. 5) the curve shows a slight upward bump just before the knee. In contrast to this, the 20 Feb. 021106 flare (Fig. 6) shows a slight downward bump just before the knee. For a sharper knee, that is for larger $\Delta \gamma$ or smaller $\Delta \epsilon$, this slight downward bump would become a negative spike.

Another discrepancy common to all flares except 20 Feb. 020958 is that the thin target $\gamma$-fit inversion is consistently greater than the numerical derivative inversion. This is because the $\chi^{2}$ fit for $\gamma$ (13) is contaminated by the thermal component which increases the value of $\gamma_{1}$. This is further aggravated by fact that low energy photon spectrum values have very small uncertainties and so are weighted heavily in the $\chi^{2}$.

\section{Discussion}

We can conclude from the results presented here that the spectral knees observed by RHESSI do not challenge the validity of thin or collisional thick target models of hard Xrays production in solar flares. This comes as no surprise for the thin target model because nothing is assumed about the acceleration or transport of the electrons. If we had found an unphysical feature in the thin target inverted electron spectrum, specifically a negative value, then the only assumptions that could be questioned are the HXR bremsstrahlung cross-section used, the bremsstrahlung process as HXR source, or that the source is optically thin.

For the collisional thick target model two important assumptions are made. Firstly, a quasi-steady state is assumed in the electron injection and loss to the thermal background. It is assumed that electrons are injected with a spectrum $F_{0}\left(E_{0}\right)$ and that all electrons subsequently lose their energy according to the same function describing energy loss per unit column depth. Secondly, for a quasi-steady state, the timescale of any variation of $F_{0}\left(E_{0}\right)$ and the life times of the electrons should be less than the integration time of the photon spectrum. Our integration times were all about $40 \mathrm{~s}$ (ten rotation of RHESSI), which is longer than the $13.7 \mathrm{~s}$ that a $100 \mathrm{keV}$ electron takes to lose all its energy in a coronal density of $10^{10} \mathrm{~cm}^{-3}$. The fact that our thick target inverted spectra contained no unphysical features therefore gives us no reason to question these assumptions concerning electron injection and transport.

There are physical processes that lead to the spectral knees even if the injected electron spectrum is a perfect power law. Firstly, we observe not only hard X-ray emission from the 

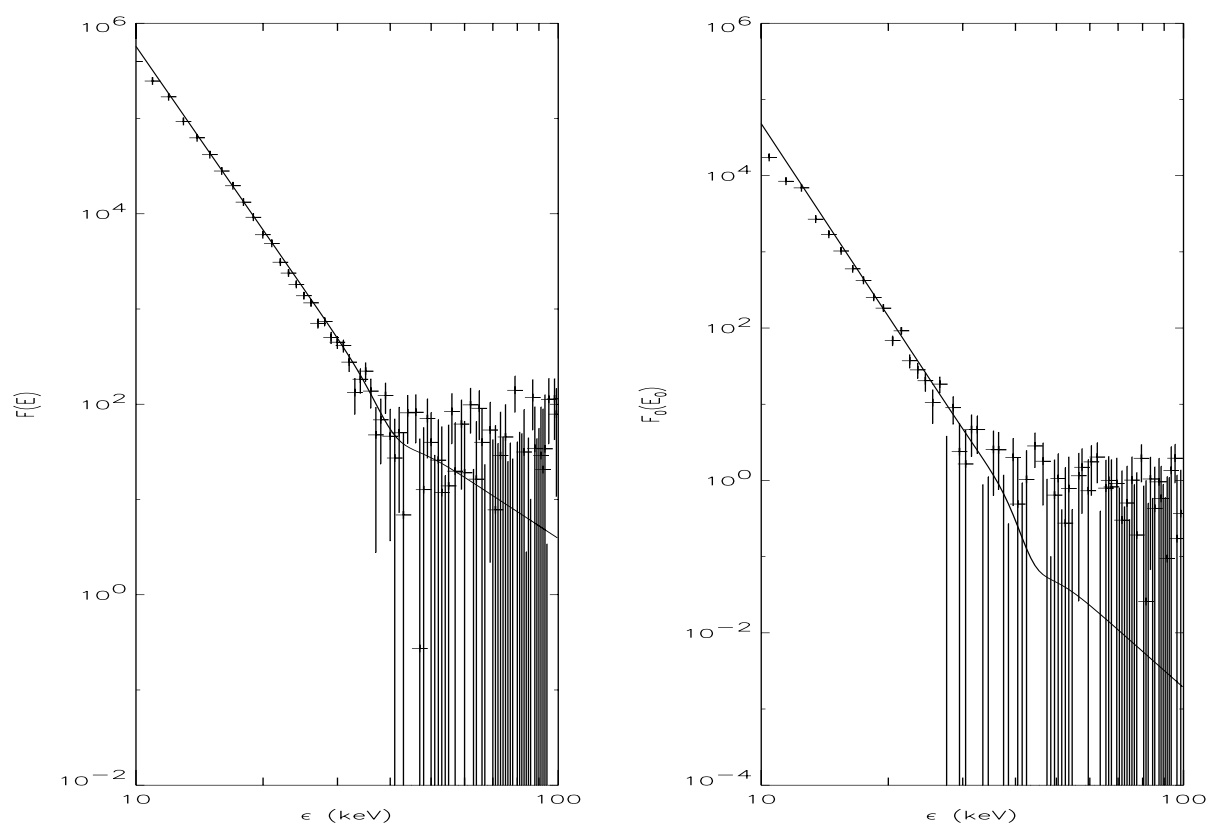

Fig. 5. The electron spectra for the 20 February 0958 UT flare. The points are a simple direct inversion and the curve is an inversion based on the parametric $\gamma$ fit.

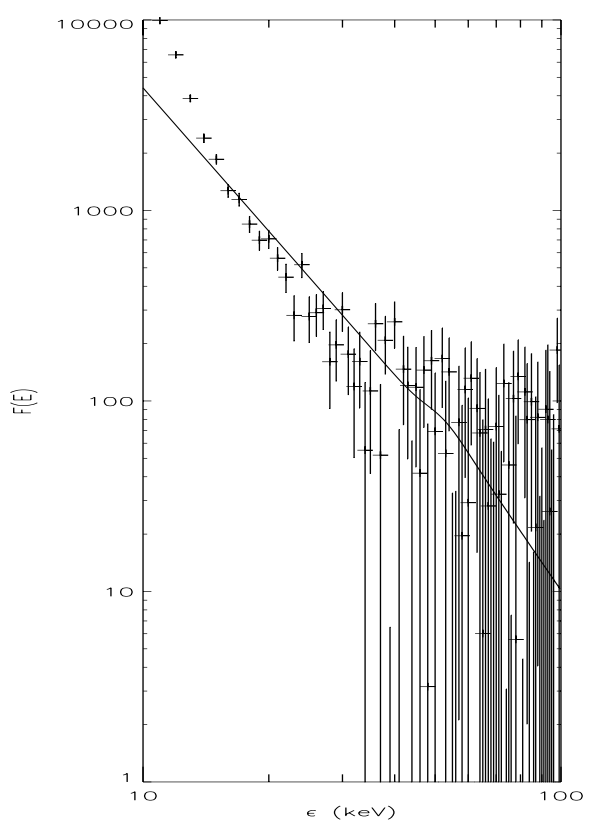

Fig. 6. The electron spectra for the 20 February 1106 UT flare.

source in the chromosphere and corona but also emission reflected from lower, denser layers of the atmosphere. Solar albedo can be up to $40 \%$ of the direct hard X-ray emission, depending primarily on the spectral index and source position; the albedo being greater for harder spectra and lower sources. Typically albedo produces deviation from power law in the range between 30-60 keV (Alexander \& Brown 2002).

Apart from albedo, the allowance for non-uniform ionisation in the chromosphere can change a spectrum significantly. The resulting spectrum has a knee in the energy range dependent on the column depth of the transition region. It was shown by Kontar et al. (2002) that effects of non-uniform ionisation can explain observable deviations from power-law in spatially

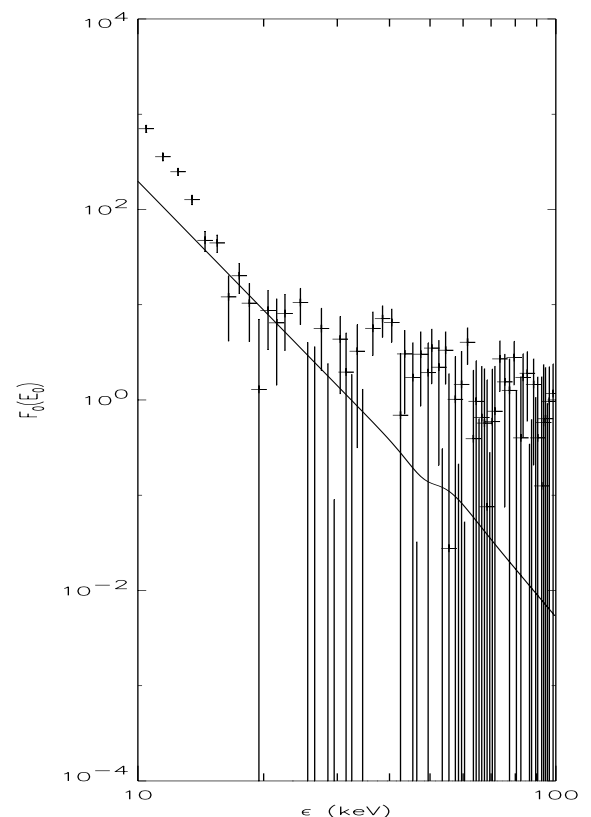

integrated RHESSI spectra. Typically, the larger flares produce higher values of plasma density as a result of chromospheric evaporation (Kontar et al. 2003) with equivalent knee energies of up to $150 \mathrm{keV}$ while smaller flares have values in the range from 30 to $60 \mathrm{keV}$. The typical change in spectral index from low to high energies is about -0.6 . Note that there is always a decrease in spectral index and so non-uniform ionisation can only explain downward knees.

Perhaps the most interesting result is the upward knee observed in the 20 Feb. 020958 UT flare. Not only was this the only example of such a knee, it was also the sharpest and largest knee (smallest $\Delta \epsilon$, largest $\Delta \gamma$ ) of the ones we studied. As discussed in Sect. 4.1.1, the large spectral index from 3 to 

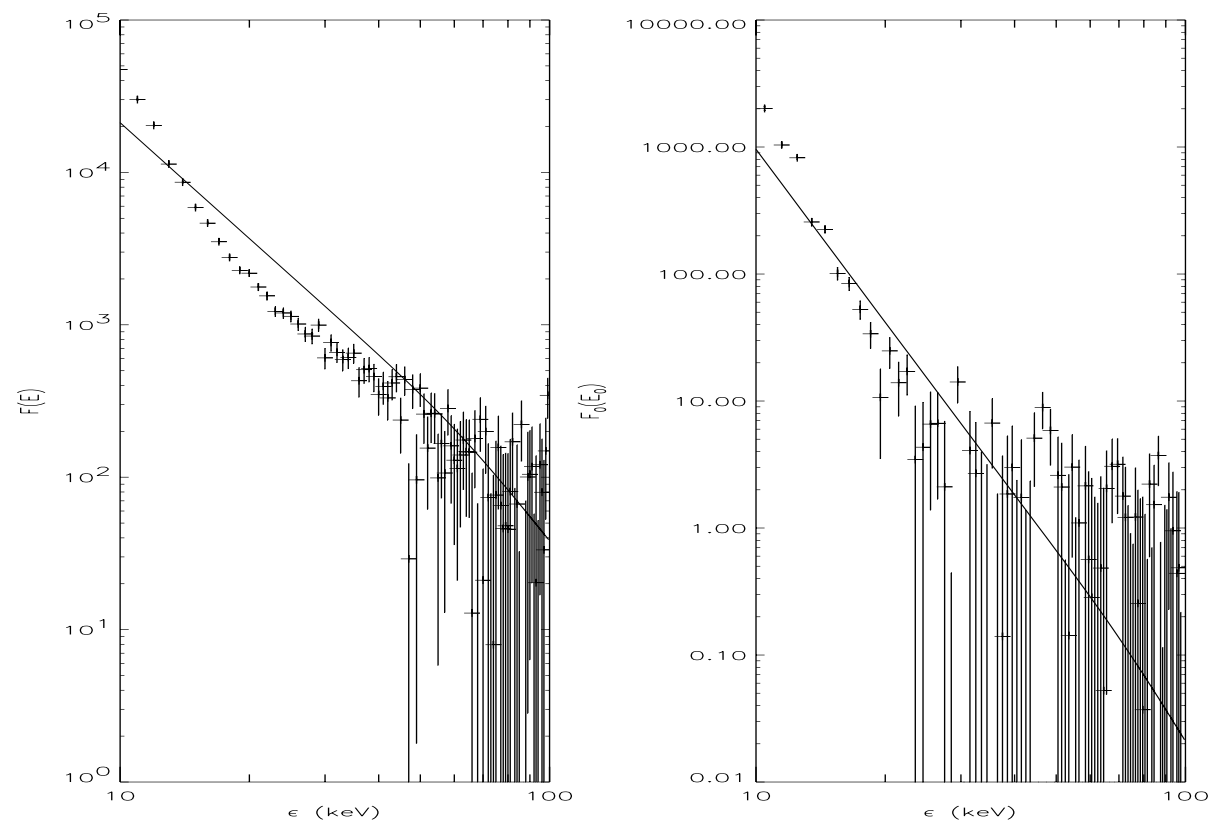

Fig. 7. The electron spectra for the 17 March 1930 UT flare.
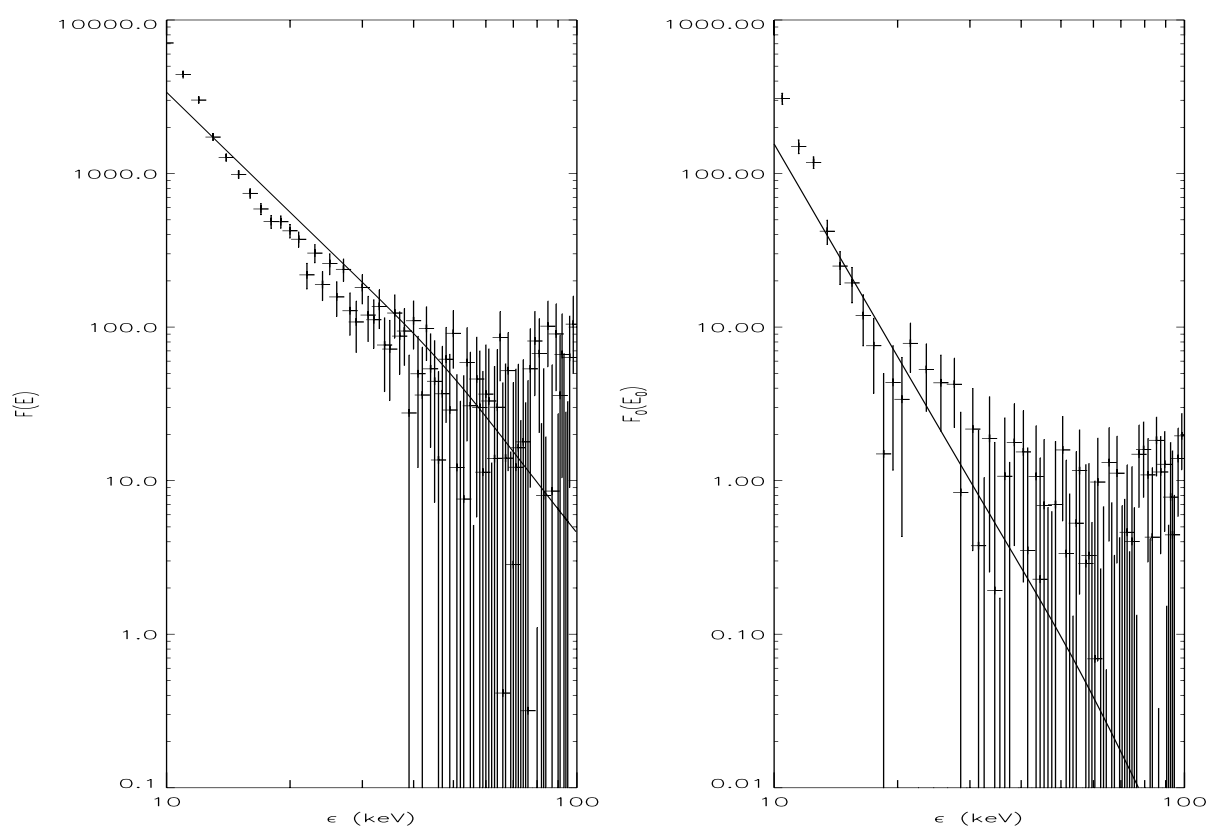

Fig. 8. The electron spectra for the 6 August 1256 UT flare.

$40 \mathrm{keV}(\gamma \sim 7)$ cannot be interpreted as being thermal because it does not decrease with increasing energy. Also, the hard spectrum $(\gamma \sim 4)$ above $40 \mathrm{keV}$ cannot be readily explained as undersubtracted background because the index is too large and it does not appear to vary (within the noise) up to $100 \mathrm{keV}$. If we accept that the knee is a real feature of the flare, then it is hard to explain it in terms of transport effects. Electrons traversing a region of non-uniform ionisation cannot explain it simply because it is an upward knee, unless $E_{*}$ is very low. Another transport explanation is that the electrons are magnetically bottled in the corona, so that the low energy electrons are in a thick target, whereas the high energy are in a thin target. However, if this were the case then we could expect a $\Delta \gamma$ no less that -2 whereas the spectra has $\Delta \gamma=-3.0$. Also, we would expect the thin-thick target explanation to produce a much more gradual knee. In the absence of any other explanations, we therefore conclude that this upward knee is most likely to be a feature of the accelerated electron distribution itself.

An important issue in the data analysis was the definition of a variable spectral index. We considered the gradient index, the gradient of the spectrum on a log-log plot, and the exponent index which measures the gradient of the line between the energy of interest and the knee energy. The advantage of the gradient index is that it is intuitive and can be applied to any form of spectrum. Its drawbacks are the large errors involved in numerical derivatives, and also that it does not generally correspond to a simple analytic form for the spectrum itself. By contrast, the exponent index can be estimated with much smaller errors 
and, by definition, it has a convenient analytic form. The exponent index's main disadvantage is the large errors and the bias introduced near the knee energy. Also, although it was not a problem in this paper, the exponent index, as we have defined it, can really only be used with knee spectra, i.e. for spectra with one localised, monotonic increasing or decreasing change in spectral index. A parametric analytic form for the exponent index $\gamma$ was used to provide thin and collisional thick target inversions. The assumption of a analytic form effectively acted as the regularisation in these inversions. However, unlike the smoothing introduced in other regularisation methods, the analytic form ensured that any negativity problems caused by the knee were not 'smoothed' away in the solution. This kind of inversion technique is therefore ideal for identifying potential unphysical features indicated by features in observed photon spectra.

Acknowledgements. A.J.C. and B.A.C.E. which to thank the Open University Research Development Fund for their support for this research. This research was partially supported by a PPARC rolling grant. The authors also thank R. Schwartz for his advice on processing the spectra.

\section{Appendix: Variable spectral indices}

In working with the theory of spectral knees there are two convenient definitions for a variable spectral index: the gradient index and the exponent index. The former is perhaps more intuitive as it is simply the gradient of the spectrum $h$ on a log-log plot:

$\gamma_{\mathrm{s}}(\epsilon)=-\frac{\mathrm{d} \ln h}{\mathrm{~d} \ln \epsilon}$.

Less intuitively, but more convenient for the theory, we can define an exponent index (the exponent of $\epsilon / \epsilon_{*}$ ) as

$\gamma(\epsilon)=-\frac{\ln h / h_{*}}{\ln \epsilon / \epsilon_{*}}$

where * denotes values at the knee. For a spectrum of the form (11), we can see that two are related by

$\gamma_{\mathrm{s}}=\gamma+\frac{\mathrm{d} \gamma}{\mathrm{d} \epsilon} \ln \frac{\epsilon}{\epsilon_{*}}$

The indices become equal in regions of constant log-log slope which means that away from the knee $\left(\left|\epsilon-\epsilon_{*}\right|>\Delta \epsilon\right)$, both will tend towards the same value. For downward knees in which the log-log slope increases monotonically we can see from (22) that $\gamma_{\mathrm{s}}<\gamma$ below the knee energy and $\gamma_{\mathrm{s}}>\gamma$ above the knee energy. These inequalities simply reverse for upward knees. If we had not defined $\epsilon_{*}$ to be the knee energy these statements would not be true and $\gamma$ and $\gamma_{\mathrm{s}}$ could differ even in regions of constant slope.

We can now compare these two definitions and examine the effect of errors on their estimates from real data by considering a spectrum of the form

$H_{i}=h\left(\epsilon_{i}\right)+\alpha_{i}$

where $i$ denotes an energy bin of average energy $\epsilon_{i}$ and $\alpha_{i}$ is the error on the true spectrum $h\left(\epsilon_{i}\right)$. The estimate of the gradient spectral index $\Gamma_{\mathrm{si}}$ can be obtained by differencing successive values. Doing so and using (23) together with realising that errors are small, i.e. $\alpha_{i} \ll H_{i}$ (true at all but the highest energies for spectra in this paper), gives following

$\Gamma_{\mathrm{si}}=-\frac{\ln h\left(\epsilon_{i+1}\right)-\ln h\left(\epsilon_{i}\right)}{\ln \epsilon_{i+1}-\ln \epsilon_{i}}-\frac{\alpha_{i+1} / h\left(\epsilon_{i+1}\right)-\alpha_{i} / h\left(\epsilon_{i}\right)}{\ln \epsilon_{i+1}-\ln \epsilon_{i}}$.

The first term is the log-log (negative) gradient between bins and the second terms is the error, which increases for decreasing separations between energy bins.

The estimate of the exponent spectral index can be written in a similar fashion by replacing $h$ with $H$ in (21)

$\Gamma_{i}=-\frac{\ln h\left(\epsilon_{i}\right)-\ln h\left(\epsilon_{*}\right)}{\ln \epsilon_{i}-\ln \epsilon_{*}}-\frac{\alpha_{i} / h\left(\epsilon_{i}\right)-\alpha_{*} / h\left(\epsilon_{*}\right)}{\ln \epsilon_{i}-\ln \epsilon_{*}}$.

The first term is the log-log (negative) gradient between the spectrum at energy bin $i$ and the knee energy. So at energies far from the knee energy, in regions where the slope is constant, $\Gamma_{i}$ will become equal (within the noise) to the gradient estimate $\Gamma_{\mathrm{si}}$. Due to the larger denominator, the errors will be smaller on the exponent index, except near the knee itself. However, the exponent index suffers from another problem in that its errors are biased by $+\alpha_{*} /\left[h\left(\epsilon_{*}\right) \ln \epsilon_{i+1} / \ln \epsilon_{*}\right]$ because the spectrum's value at the knee energy is used for estimating $\Gamma_{i}$ for every $i$. This bias reverses over the knee which means that the overall change in gamma, $\Delta \gamma$, will also be biased. This effect of the bias, which is not large, can be seen most clearly in Fig. $1 b$. The presence of the bias will be most pronounced when $\epsilon_{i} \sim \epsilon_{*}$ and so spikes are seen near the energy in plots of $\Gamma_{i}$ (Figs. $1 \mathrm{~b}$ to $4 \mathrm{~b}$ ). The fundamental reason for this problem is that the assumed form for $\gamma(11)$ permits $\gamma(\epsilon)$ to take any finite value.

\section{References}

Alexander, R. C., \& Brown, J. C. 2002, Sol. Phys., 210, 407

Brown, J. C. 1971, Sol. Phys., 18, 489

Brown, J. C., \& MacKinnon, A. L. 1985, ApJ, 292, L31

Brown, J. C., \& Emslie, A. G. 1987, ApJ, 331, 554

Brown, J. C., MacKinnon, A. L., van den Oord, G. H. J., \& Trottet, G. 1991, A\&A, 242, 13

Brown, J. C., McArthur, G., Barrett, R. K., McIntosh, S. W., \& Emslie, A. G. 1998, Sol. Phys., 179, 379

Haydock, E. L., Brown, J. C., Conway, A. J., \& Emslie, A. G. 2001, Sol. Phys., 203, 355

Emslie, A. G. 1978, ApJ, 224, 241

Emslie, A. G., Barrett, R. K., \& Brown, J. C. 2001, ApJ, 557, 921

Johns, C. M., \& Lin, R. P. 1992, Sol. Phys., 137, 121

Kontar, E. P., Brown, J. C., \& McArthur, G. K. 2002, Sol. Phys., 210, 419

Kontar, E. P., Emslie, A. G., Brown, J. C., et al. 2003, ApJL, submitted Lin, R. P., \& Hudson, H. S. 1976, Sol. Phys., 50, 153

Lin, R. P., \& Schwartz, R. A. 1987, ApJ, 312, 462

Lin, R. P., Dennis, B. R., \& The RHESSI team 2002, Sol. Phys., 210, 3

McArthur, G. K. 2000, Ph.D. Thesis, University of Glasgow

Piana, M. 1994, A\&A, 288, 949

Piana, M., Brown, J. C., \& Thompson, A. M. 1995, A\&A, 156, 315

Piana, M., \& Brown, J. C. 1998, A\&A, 132, 291

Piana, M., Barrett, R. K., Brown, J. C., \& McIntosh, S. W. 2001, Inverse Problems, 15, 1469

Thompson, A. M., Brown, J. C., Craig, I. J. D., \& Fülber, C. 1992, A\&A, 265, 278

Vilmer, N., Krucker, S., Lin, R. P., \& The RHESSI team 2002, Sol. Phys., 210, 261 